\title{
Particle methods in natural science and engineering
}

\author{
Christian Holm ${ }^{1, a}$, Thomas Ertl ${ }^{2, b}$, Siegfried Schmauder ${ }^{3, c}$, Johannes Kästner ${ }^{4, d}$, \\ and Joachim Gross ${ }^{5, e}$ \\ ${ }^{1}$ Institute for Computational Physics, University of Stuttgart, Stuttgart, Germany \\ ${ }^{2}$ Visualization Research Center, University of Stuttgart, Stuttgart, Germany \\ ${ }^{3}$ Institute for Materials Testing, Materials Science and Strength of Materials, University \\ of Stuttgart, Stuttgart, Germany \\ ${ }^{4}$ Institute for Theoretical Chemistry, University of Stuttgart, Stuttgart, Germany \\ ${ }^{5}$ Institute of Thermodynamics and Thermal Process Engineering, University of Stuttgart, \\ Stuttgart, Germany
}

Received 23 January 2019

Published online 8 March 2019

\begin{abstract}
This special topics issue offers a broad perspective on the recent developments and reviews of state-of-the-art particle methods in science and engineering applications. This issue grew out of contributions delivered during the closing conference of the Collaborative Research Centre (CRC) 716 that for the last twelve years was contributing important works to the area of "dynamical simulations of systems with large particle numbers". Since particle methods are present in many fields of science and engineering, the papers collected here span a considerable range of subjects and questions, but they also illustrate numerous connections to both fundamental science and technological/industrial applications. In addition reviews display the current state of three software packages that have been developed in this $\mathrm{CRC}$ in the area of simulations and visualization.
\end{abstract}

Computer simulations are by now an established scientific field that is used to bridge the gap between experimental data and a purely theoretical analytical explanation. Especially useful are particle-based molecular dynamics (MD) simulations since from an accurate description of the pair interactions of microscopic particles it is possible to deduce the macroscopic material behavior if a sufficiently large ensemble can be simulated for long enough times to deduce equilibrium properties without finite-size effects.

The notion of a particle can be manifold. A particle can denote a pseudo-particle in electronic structure theory, an atom, a molecule, or the monomer of a polymer. In even more coarse-grained (CG) approaches they can also represent an effective agglom-

\footnotetext{
a e-mail: holm@icp.uni-stuttgart.de

b e-mail: thomas.ertl@vis.uni-stuttgart.de

c e-mail: siegfried.schmauder@imwf.uni-stuttgart.de

d e-mail: kaestner@theochem.uni-stuttgart.de

e e-mail: joachim.gross@itt.uni-stuttgart.de
} 
erate of particles, a lump of fluid, a grain of sand, a planet, or even a galaxy. Particle methods can bridge the scales between quantum chemical ab-initio approaches and electronic density functional theories up to the macroscopically valid continuum theories that solve suitably constructed differential equations via finite-element or finite-volume, or other suitable schemes. The very beneficial linear scaling properties of molecular dynamics simulations with system size have allowed to simulate always larger systems by simply waiting for the next hardware revolution. In fact, the system sizes in the last sixty years could grow exponentially due to the exponential increase in computing power that the industry has provided, and that is well represented by Moore's law even up to now. Nevertheless, it was not only the mere speed increase of computers that has led to many new discoveries, but also the employed algorithms have evolved in the last seventy years since the second half of the twentieth century. That period saw basically the rise of computers to support scientists in solving complex equations and to advance our understanding in many areas of science and technology. The increase in system size and simulation time has gone hand in hand with a tremendous increase in data that have to be analyzed properly. Large scale visualization and data reduction techniques have therefore become vital for handling this "big data".

The collaborative research centre (CRC) 716 was established in January 2007 by the German Science Foundation (DFG) with the topical theme "Dynamical Simulations of Systems with Large Particle Numbers". It was funded with over 1.5 million Euro per year combining roughly 20 research groups working at the University of Stuttgart in various faculties ranging from chemistry over mathematics and physics to computer science and electrical engineering, energy technology, chemical engineering and biotechnology, up to aerospace engineering and engineering design, production design and automotive engineering, and also invoking the high-performance computing facility in Stuttgart, the HLRS, and the visualization research centre (VISUS). Established under the leadership of H. Hasse and T. Ertl, followed by H.-R. Trebin, and finally by C. Holm, the CRC 716 has produced impressive results during the last twelve years. This special issue documents some of the latest results, and gives as well some overviews of topcis and the standing in the scientific world. It also contains three papers by external researchers that have been either formally associated with the CRC, or have been accompanying the CRC as external referees.

The twenty-three papers of this issue can be roughly divided into five major categories, block A on problems in mechanics and thermodynamics, the block B about problems in material science, the block $\mathrm{C}$ about biochemical and biophysical problems, and the block D dealing with scalable visualization algorithms and efficient implementations of simulation algorithms. In the last block $\mathrm{E}$ we have included the state-of-the-art descriptions of three software packages that were significantly extended during the installment of the $\mathrm{CRC}$, and that have contributed largely to the success of the CRC 716. These are the ESPResSo package (http://www. espressomd.org/), the IMD package (http://imd-doc.sourceforge.net/wiki/ doku.php/), and the MegaMol visualization framework (http://megamol.org/). The wide range of topics and the highly interdisciplinary approach we undertook in many projects have led to very interesting, and sometimes even groundbreaking research papers. In the following we will describe briefly the content of each paper, and how it fits into the overarching scheme.

We begin with the papers of block A on engineering questions in thermodynamics and mechanics. The first contribution of Hopp-Hirschler et al. [1] is concerned about an incompressible version of the smoothed particle hydrodynamics method (SPH), the so-called ISPH. The authors present a first-order implicit time stepping scheme for ISPH to eliminate the viscous time step criterion. They study Poiseuille flow, Taylor- Green flow and Rayleigh-Taylor instability and compare the results 
of traditional semi-implicit and implicit schemes to ISPH. Energy conservation is investigated to highlight differences between the approaches. In the contribution of Smiljanic et al. [2] a coarse-graining approach for the soot agglomeration of nanoparticles is presented. In the article collision frequencies of CG simulations are compared to equivalent Langevin dynamics simulations where all nano-sized particles are tracked. The results demonstrate the applicability of the CG procedure. This simplification significantly reduces the number of degrees of freedom and allows for the computation of much larger systems and for better collision statistics of larger clusters. The contribution of Baz et al. [3] deals with the parameterization of forcefields for accurate MD simulations, and how to address the problem of overfitting. In particular, the authors investigate the question whether dynamic properties can be used to discriminate among different sets of force-field parameter that work equally well for static thermodynamic properties. The authors study a model of 1-propanol where united-atom sites are described through Mie potentials and point charges. Four models were investigated with the results that the degeneracy of force field parameters observed in the static properties was also observed in the dynamic properties. The authors propose therefore to consider homologous series in order to define a less degenerate optimization problem. The contribution of Garcia and Hasse [4] deals with the well-known sampling problem that is encountered when a high-molecular weight polymeric system in water is studied fully atomistically. As test case they study the case poly(n- isopropylacrylamide) (PNIPAM) in water. Although the polymer is only thirty monomers long, and the simulation time was one microsecond, their careful analysis revealed equilibration times of the order of 600-700 ns, concluding that even such a long time is way too short to sample equilibrium states of polymers in solution with direct MD simulations despite the increasing available computing power. The authors conclude that enhanced sampling techniques and workarounds, such as simplified scenarios or CG models, will be necessary.

The next block $\mathrm{B}$ is dealing with material science problems. In the paper by Hocker at al. [5] a combination of MD and the Metropolis Monte Carlo (MMC) method was employed in order to study the formation of $\mathrm{Ni}_{3} \mathrm{Si}$ precipitates with $\mathrm{L}_{2}$ crystal structure. Since $\mathrm{L} 1_{2}$ structured precipitates are very important for strengthening of several alloys, planar defects and dislocations in $\mathrm{Ni}_{3} \mathrm{Si}$ and $\mathrm{Ni}_{3} \mathrm{Al}$ were studied. Furthermore, the consequences of dislocations were discussed and interactions of edge dislocations with $\mathrm{Ni}_{3} \mathrm{Si}$ and $\mathrm{Ni}_{3} \mathrm{Al}$ precipitates were studied and rationalised from the analysis of charge densities. The work of Eisfeld et al. [6] describes a simple description to compute the electronic thermal conductivity of aluminum in the crystalline solid as well as the liquid phase over a wide range of temperatures and densities in the crystalline solid as well as the disordered liquid phase. All calculations are based on first order perturbation theory and the pseudo-potential theory without resorting to ab-initio simulations. Wherever possible, the results are compared to experimental data or ab-initio MD simulations. In addition a straightforward approach is suggested to estimate the complex permittivity from the Drude model. The contribution by Hertkorn et al. [7] performs a comparative analysis of defect centers in nano-scale spherical diamonds. They focussed on the well-known negatively charged nitrogenvacancy defect center and the two neutral silicon- and germanium-vacancy centers in nanodiamonds. Quantum mechanical simulations based on density functional theory are performed in order to assess the influence of the surface termination and the defect type in the electronic structure of the defective nanodiamonds. In summary their work provides a comparative analysis of the termination effect on the three types of vacancy defect centers for their deeper understanding in view of their technological relevance. In the paper of Copplestone et al. [8] the laser-plasma interaction is simulated via three-dimensional Particle-In-Cell methods. They employ high-order methods for drastically reducing the required number of degrees of freedom while still 
capturing the complex physical nature of non-linear processes. The suitability of highorder methods for the acceleration of protons from thin films by intense short-pulse lasers in terms of accuracy and efficiency is demonstrated in their paper. Bauerhenne and Garcia [9] study the laser light interaction of femtosecond laserpulses with silicon via computer simulations. They convincingly demonstrate that a potential for Si at high electronic temperatures $T_{\mathrm{e}}$, which was developed from ab-initio MD simulations performs much better when compared to other available $T_{\mathrm{e}}$-dependent $\mathrm{Si}$ potentials, and to some widely used ground state $\mathrm{Si}$ potentials, which were adapted to nonequilibrium by fitting to ab-initio MD simulations. Their proposed potential yields the best description since its analytical shape was optimized for the ground and the laser excited state.

Block $\mathrm{C}$ deals with biochemical and biophysical problems. The paper by Ferrario and Pleiss [10] describes molecular simulations of enzymes under non-natural conditions. This is important since the development of novel sustainable biocatalytic processes requires systematic and comprehensive tools for engineering, integrated into a framework for the simultaneous optimizations of enzyme, substrate, solvent, and reaction conditions. The authors describe the interaction of the substrate with the solvent by its activity coefficient, whereas the interaction of the substrate with the protein and substrate access to the active site were characterized by a binding free energy along a reaction coordinate, and the protein-solvent interaction was modeled by a Langmuir model. The MD simulation of protein aggregation identified a delicate balance of kinetics and thermodynamics of competing contacts during the nucleation process. The contribution of Weik et al. [11] describes an extended mean-field model for solving the electrokinetic equations of a dsDNA confined to a structureless cylindrical pore. This is done by incorporating a suitably constructed friction term into the Nernst-Planck equation whose importance has been discovered by performing simulations including the solvent on an all-atom level. Solving the modified electrokinetic equations using the finite element method, they demonstrate that this model is able to reproduce experimental and atomistic MD results for dsDNA current modulations. This model allows for a fast and accurate evaluation of new geometric arrangements of the DNA within the pore at little computational costs. Alvarez-Barcia and Kästner [12] investigated if and how copper can be accommodated in the active site of the enzyme FGE that is a copper and oxygen-dependent protein, which catalyses $\mathrm{C}-\mathrm{H}$ activation. Using a hybrid quantum mechanics/molecular mechanics (QM/MM) approach the authors show how copper can be incorporated in the enzyme based on two crystal structures containing $\mathrm{Ag}(\mathrm{I})$ and $\mathrm{Cd}(\mathrm{II})$ in place of $\mathrm{Cu}(\mathrm{I})$ and $\mathrm{Cu}(\mathrm{II})$. They discovered that both, $\mathrm{Cu}(\mathrm{I})$ and $\mathrm{Cu}(\mathrm{II})$, can be accommodated with minimal structural rearrangements. This structural flexibility may allow the enzyme to catalyze the redox process and accommodate copper in both oxidation states. The paper by Oprzeska-Zingrebe et al. [13] uses the framework of KirkwoodBuff (KB) theory to rationalize the destabilizing influence of ectoine on DNA hairpin structures in aqueous solutions. Their analysis of the individual contributions with regard to Kirkwood-Buff integrals reveals an outstanding importance of the co-solute binding behavior to the individual native and unfolded DNA hairpin conformations. In combination with transfer free energies, they showed that the binding behavior of the co-solutes also changed the unfolding free energy difference. Moreover, a thermodynamic cycle to evaluate several transfer free energy differences in terms of single preferential binding coefficient is presented. Further, the authors give a nice review of the usefulness of KB theory. In their contribution Maier et al. [14] investigate the potential and inherent characteristics of diamondoid-functionalized electrodes, which can be embedded in sensing nanopores, using quantum-mechanical density functional calculations. The authors first investigated the interaction details of memantine with the DNA nucleotide units. In a second step they placed nucleotides 
within the nanogap formed by the diamondoid-functionalized electrodes. Quantum transport calculations showed a high sensitivity of the electrodes in distinguishing among the different nucleotide types. The effect of an aqueous environment was also included and the dynamic behavior of the conductance across the functionalized electrodes was addressed. In the last contribution to C-block Kandzia and Zacharias [15] studied the dynamics of a full-length yeast Hsp90 dimer using all-atom MD simulations in explicit solvent. The Hsp90 chaperone is a complex homodimeric biological assembly that assists in the folding of proteins. The simulations were started from yeast Hsp90 in two different conformational states resembling the open (ADPbound) and closed (ATP-bound) states of the chaperone complex. On the simulated time scale of $300 \mathrm{~ns}$ no significant dependence of the bound nucleotide and also no significant dependence on a W585T substitution was observed. The analysis of conformational fluctuations indicated only modest differences in local fluctuations of the monomers for open versus closed state and the global dimer changes were mediated by only small local motions of the C-terminal Hsp90 segments.

In block $\mathrm{D}$ visualization techniques and efficient implementations of algorithms are described. In their mini-review Reina et al. [16] put the last twelve years of atomistic particle-based visualization research in context with their own efforts within the CRC 716. They concentrate on the visualization and analysis of particle-based data sets, and answer the question on how to bring these visualizations to the workstation of domain scientists without the need for a large rendering infrastructure. They discuss how their decisions and goals evolved over time and present selected success stories. They conclude with an outlook on the challenges that still require additional research and to which extent the requirements and constraints of the current research have changed the way visualizations work. In the next mini-review Schatz et al. [17] describe the state-of-the-art method of interactive visualization of complex properties and the dynamics of biomolecules and put their respective work performed during the last twelve years within the CRC 716 in context with the visualization community. Molecular visualization is one of the oldest branches of scientific visualization, yet it has advanced tremendously during the last two decades, mostly due to the constant improvement of computing hardware, and here especially the advent of freely programmable GPUs. Their review includes advances in the field of molecular surface computation and rendering, interactive extraction of protein cavities, and comparative visualization for biomolecules. The main research focus was on the development of methods that assist the interactive and explorative visual analysis of large, dynamic molecular data sets on single desktop computers, often with GPU-accelerated algorithms. In the contribution of Zhou and Weiskopf [18] methods for interactive particle rendering techniques, multi-view particle visualization systems, multivariate visualization techniques, and methods for correlation visualizations are reviewed that are vital for gaining insight into particle data. The visualization of multivariate particle data is typically performed in multiple linked view systems that render particles of interest, which are selected by the user interactively with brushing-and-linking. To this end, the non-spatial aspects of particles are explored with multivariate visualization methods, e.g., scatter plots, scatter plot matrix, parallel coordinates, dimensional reduction and radial plots. In their tutorial review paper Mehl and Lahnert [19] describe a minimally invasive approach for integrating dynamically adaptive tree-structured grids into existing simulation software that has been developed for regular Cartesian grids. The authors introduce different physical models that span a wide range of typical simulation characteristics: from grid-based lattice-Boltzmann, finite-volume and finite-difference discretized models to particle-based molecular dynamics models. They extended the grid framework p4est to give random access to direct neighbors in order to keep application code changes as light-weight as possible. Besides the option to use adaptive grids, and, therewith, 
simulate substantially larger spatial domains and time spans, the application code ESPResSo in their example also profits from functionalities such as domain partitioning and inter-process communication in the parallel implementation, that previously had to be provided by the application code itself and can now be taken care of (in an optimized way) by the grid framework. Their results show the general suitability and potential of their approach for various show-cases. The last contribution of Hirschmann et al. [20] to the D-block deals with the task to implement an unstructured domain decompositions which is useful for inhomogeneous short-range MD in the ESPResSo package. This is important particularly for inhomogeneous particle distributions that dynamically change over time and that require flexible load-balancing methods to achieve good parallel efficiency, like the simulations described in the work of Smiljanic et al. [2]. The authors have implemented a general framework that can support different load-balancing methods and that can extend existing simulation packages in a minimally invasive way. A new graph-based partitioning strategy is presented that leads to unstructured spatial domain decompositions and which integrates well into the existing framework. For several load metrics, graph partitioning leads to better results than space-filling curves. The results indicate that the parallel performance for a given scenario requires a delicate combination of partitioning strategy and load metrics.

In the last block E the current state of three open source software packages, that experience continuous improvements and that have been largely extended during the funding time of the CRC 716 is described.

These are in particular, the contribution by Weik et al. [21] about the Extensible Simulation Package for Research on Soft Matter (ESPResSo, http://www . espressomd.org), that has been considerably enhanced in the last ten years towards engineering applications like soot aggregation, catalysis, porous materials, active matter, and many more. The new version 4.0 now comes with a Python interface, making it even more versatile and flexible. In addition, many new simulation methods have been implemented. The ITAP Molecular Dynamics (IMD, http: //imd-doc.sourceforge.net/wiki/doku.php) package [23] is a simulation package designed for large-scale simulation studies in materials sciences. IMD can be run with a large number of effective many-body interactions that can be deduced from ab-initio calculations. Here Roth et al. describe the design and capabilities of IMD, the interaction of IMD with other codes, and put a special focus on laser ablation problems. The contribution of Gralka et al. [22] describes the software MegaMol (http://megamol.org), a low-overhead prototyping framework for interactive visualization of large scientific data sets, like the ones produced by molecular dynamics simulations. MegaMol has originally been developed to support the visualization and analysis of particle-based data sets but meanwhile new algorithms and techniques have been implemented to handle many more diverse tasks, such as information visualization. Additionally, improvements have been made on the software engineering side to make MegaMol more accessible and easy to handle.

To conclude, the entire issue demonstrates the breadth of research topics where particle methods can be used, and to which level of complexity visualization tools and simulation algorithms have meanwhile been developed. Nevertheless, there are still many open research questions, and we undoubtedly see more advances in simulation science in the near future. We hope the readers of this issue find these contributions inspiring and helpful for their own research in engineering and natural science applications. At the end, we would like to take the opportunity to thank the German Research Foundation (DFG) at this point for their generous support for the last twelve years, our referees for their helpful criticisms, an the University of Stuttgart for additional basic funding. 


\section{References}

1. M. Hopp-Hirschler, U. Nieken, Eur. Phys. J. Special Topics 227, 1501 (2018)

2. M. Smiljanic, R. Weeber, D. Pflüger, C. Holm, A. Kronenburg, Eur. Phys. J. Special Topics 227, 1515 (2018)

3. J. Baz, N. Hansen, J. Gross, Eur. Phys. J. Special Topics 227, 1529 (2018)

4. E.J. García, H. Hasse, Eur. Phys. J. Special Topics 227, 1547 (2018)

5. S. Hocker, H. Lipp, S. Schmauder, Eur. Phys. J. Special Topics 227, 1559 (2018)

6. E. Eisfeld, H.-R. Trebin, J. Roth, Eur. Phys. J. Special Topics 227, 1575 (2018)

7. J. Hertkorn, J. Wrachtrup, M. Fyta, Eur. Phys. J. Special Topics 227, 1591 (2018)

8. S.M. Copplestone, M. Pfeiffer, S. Fasoulas, C.-D. Munz, Eur. Phys. J. Special Topics 227, 1603 (2018)

9. B. Bauerhenne, M.E. Garcia, Eur. Phys. J. Special Topics 227, 1615 (2018)

10. V. Ferrario, J. Pleiss, Eur. Phys. J. Special Topics 227, 1631 (2018)

11. F. Weik, K. Szuttor, J. Landsgesell, C. Holm, Eur. Phys. J. Special Topics 227, 1639 (2018)

12. S. Alvarez-Barcia, J. Kästner, Eur. Phys. J. Special Topics 227, 1657 (2018)

13. E.A. Oprzeska-Zingrebe, M. Kohagen, J. Kästner, J. Smiatek, Eur. Phys. J. Special Topics 227, 1665 (2018)

14. F.C. Maier, C.S. Sarap, M. Dou, G. Sivaraman, M. Fyta, Eur. Phys. J. Special Topics 227, 1681 (2018)

15. F. Kandzia, M. Zacharias, Eur. Phys. J. Special Topics 227, 1693 (2018)

16. G. Reina, P. Gralka, T. Ertl, Eur. Phys. J. Special Topics 227, 1705 (2018)

17. K. Schatz, M. Krone, J. Pleiss, T. Ertl, Eur. Phys. J. Special Topics 227, 1725 (2018)

18. L. Zhou, D. Weiskopf, Eur. Phys. J. Special Topics 227, 1741 (2018)

19. M. Mehl, M. Lahnert, Eur. Phys. J. Special Topics 227, 1757 (2018)

20. S. Hirschmann, C.W. Glass, D. Pflüger, Eur. Phys. J. Special Topics 227, 1779 (2018)

21. F. Weik, R. Weeber, K. Szuttor, K. Breitsprecher, J. de Graaf, M. Kuron, J. Landsgesell, H. Menke, D. Sean, C. Holm, Eur. Phys. J. Special Topics 227, 1789 (2018)

22. P. Gralka, M. Becher, M. Braun, F. Frieß, C. Müller, T. Rau, K. Schatz, C. Schulz, M. Krone, G. Reina, T. Ertl, Eur. Phys. J. Special Topics 227, 1817 (2018)

23. J. Roth, E. Eisfeld, D. Klein, S. Hocker, H. Lipp, H.-R. Trebin, Eur. Phys. J. Special Topics 227, 1831 (2018) 